\title{
PARTICIPACIÓN COMUNITARIA EN EL MODELO DE SALUD DEL PUEBLO INDÍGENA DE RAMA CAY, BLUEFIELDS ${ }^{16}$
}

\author{
Mario Guillermo Mejía López ${ }^{17}$
}

\section{RESUMEN}

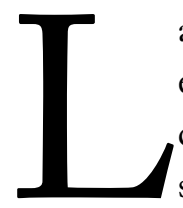

a participación comunitaria aplicada al campo de la salud puede entenderse como los diferentes alcances en los programas, referidos a la planificación, evaluación, control de acciones y servicios de salud hacia la comunidad. Es decir, la comunidad participa en la identificación del problema, en la toma de decisiones, co-gestión y autogestión de programas y servicios de salud.

Este estudio tiene como propósito describir los niveles de participación de los comunitarios de Rama Cay en materia de salud pública, de tal forma que se revitalicen los aportes del pueblo indígena Rama a la práctica de la medicina tradicional desde su propia dinámica de vida. Es importante mencionar que el pueblo Rama es el pueblo menos numeroso en las regiones autónomas de la Costa Caribe nicaragüense..

El presente trabajo fue de carácter participativo, en el mismo se estableció un dialogo de saberes entre funcionarios del Ministerio de Salud, hombres y mujeres comunitarios de Rama Cay, médicos tradicionales, líderes de la Junta Comunal y promotores de salud con presencia en la comunidad. Por tanto, para conocer la participación comunitaria en la construcción de un modelo de salud para los Rama se dimensionaron los planos de la salud individual y comunal.

En el plano de la salud individual las enfermedades de tipo biológico y físico son una prioridad. La comunidad es consciente de las limitaciones del servicio de salud que ofrece el Centro de Salud y de las alternativas que existen en el campo de la etnomedicina, así mismo, de la coexistencia de dos modelos de atención a la

16 Estudio desarrollado por el Instituto de Medicina Tradicional y Desarrollo Comunitario (IMTRADEC) Recinto Bluefields.

17 Licenciado en Sociología con mención en Autonomía. 
salud que solucionan sus distintos problemas. Por otro lado, en relación a la salud comunitaria la comunidad de manera general asiste al centro de salud en donde los atiende personal del Ministerio de Salud; sin embargo, la comunidad también cuenta con parteras, curanderos, brigadistas, promotores.

Los líderes y los comunitarios consideran que el trabajo de la Junta Comunal, especialmente en el sector salud es pasivo. Es decir, en relación a la salud comunitaria, la junta comunal no tienen funciones asignadas, solamente realizan actividades enfocadas hacia la limpieza comunal y jornadas de aseo. Así mismo, el apoyo del Ministerio de Salud es limitado y cerrado debido a su estructura y política de gerencia.

\section{PRESENTACIÓN}

En Nicaragua durante la década de los 80's, el gobierno Sandinista estableció un sistema único de salud, el servicio era gratuito e incluía la donación de medicinas recetadas al paciente. Con el cambio del gobierno en 1990, desapareció no solo el sistema único de salud, también la gratuidad del servicio. En el ámbito del servicio de salud se pasó a un nuevo esquema, estableciendo un Sistema Local de Atención Integral a la Salud (SILAIS) por cada departamento, con excepción del departamento de Managua, que tiene actualmente dos, y las dos Regiones Autónomas que mantuvieron el esquema de atención regional de salud.

En esta nueva concepción de salud pública se estableció como el responsable de la definición de políticas, normas, planes y programas de salud pública, así como de la supervisión de todo el sistema. Pero además, en este se introdujo como nueva política: el pago de los servicios, dejando la gratuidad para los casos de los sectores más vulnerables.

A nivel nacional y regional las principales limitaciones del sector salud son: el déficit de personal médico y paramédico en algunas de sus unidades de salud, el insuficiente abastecimiento de medicinas y presupuesto inadecuado para el mantenimiento correctivo y preventivo de las unidades de salud y la rotación de personal médico en servicio social. Por otro lado, hay que mencionar la estructura vertical del sistema de salud que brinda servicios sin la participación de las estructuras comunitarias.

Por lo anterior, este estudio tiene como propósito describir los niveles de participación de los comunitarios de Rama Cay en materia de salud publica, de tal forma que se revitalicen los aportes del pueblo indígena Rama en Rama Cay sobre la practica de la medicina tradicional desde su propia cosmovisión. 


\section{ASPECTOS HISTÓRICOS Y CONCEPTUALES}

El proceso de unificación del territorio de lo que hoy son las Regiones Autónomas de la Costa Caribe en el Estado de Nicaragua, inició de forma tardía a finales del siglo pasado, décadas después de la independencia de Nicaragua del imperio Español. Esta vasta región de Nicaragua ha pasado por una historia colonial distinta del resto del país en la cual se destaca la influencia británica (Conferencia internacional del grupo consultivo sobre la reconstrucción y transformación de América Central, 1999).

Debido a esta historia que ha marcado diferencias entre el Atlántico y el Pacífico, y a la influencia británica en la Costa Caribe totalmente distinta del resto del territorio nicaragüense, se formó una sociedad pluricultural y multilingüe, compleja y dinámica, resultando en la conservación de idiomas y culturas de los pueblos indígenas. También, es importante mencionar el traslado forzado de poblaciones afro descendientes de sus territorios nativos en la época colonial; y de inmigraciones posteriores de poblaciones mestizas, provenientes del Pacífico a lo largo del presente siglo (Ibíd.).

Los Rama pertenecen al pueblo menos numeroso de todos los pueblos indígenas y comunidades étnicas de la Costa Caribe. Constituyen apenas el $0.55 \%$ de la población regional (González, 2001). La mayoría están ubicados en la isla de Rama Cay, conformando una comunidad indígena la que se define como "la colectividad humana que mantiene una continuidad histórica con las sociedades anteriores a la colonia, cuyas condiciones sociales, culturales y económicas les distingue de otros sectores de la sociedad nacional y que están regidos total o parcialmente por sus propias costumbres y tradiciones ${ }^{18}$ " (Asamblea Nacional de la República de Nicaragua, 2002).

Para obtener un concepto operativo sobre la comunidad Rama, la definiremos como "el conjunto de familias de ascendencia amerindia establecidas en espacio territorial, que comparten sentimientos de identificación, vinculados al pasado aborigen de su pueblo indígena, y que mantienen una identidad y valores propios de una cultura tradicional, así como sus formas de tenencia y uso comunal de tierras y de una organización propia” Ibíd.

Aguilar Idánez (sf) define de manera práctica a la comunidad como "una agregación social o conjunto de personas que, en tanto habitan un espacio geográfico delimitado y delimitable, operan en redes de comunicación dentro de la misma, pueden compartir equipamientos y servicios comunes, y desarrollan un sentimiento de pertenencia o identificación con algún símbolo local; y, como consecuencia de ello, puede desempeñar funciones sociales a nivel local, de tipo económico, de socialización, de control social, de participación social y de apoyo mutuo" (Aguilar, s.f. Participación ciudadana en salud, evaluación de experiencias)

18 Asamblea Nacional de la República de Nicaragua. 2002 en: Ley del Régimen de Propiedad Comunal de los Pueblos Indígenas y Comunidades Étnicas de las Regiones Autónomas de la Costa Atlántica de Nicaragua y de los Ríos Bocay, Coco, Indio Y Maíz. 
La participación comunitaria puede entonces entenderse como el proceso social en virtud del cual grupos específicos, que comparten alguna necesidad, problema o centro de interés y viven en una misma comunidad, tratan activamente de identificar estas necesidades, problemas o centros de interés, adoptan decisiones y establecen mecanismos para atenderlas. Aplicado al campo sanitario, la participación comunitaria puede entenderse con alcances diferentes: como participación en los beneficios, esfuerzos, evaluación, control, planificación, programación de acciones y servicios sanitarios. Como participación en la identificación del problema, en la toma de decisiones, cogestión y autogestión de programas y servicios de salud (Ibíd.)

\section{METODOLOGÍA}

El presente trabajo es de carácter participativo, se estableció un diálogo de saberes entre funcionarios del Ministerio de Salud, hombres y mujeres comunitarios de Rama Cay, médicos tradicionales, líderes de la Junta Comunal y promotores de salud con presencia en la comunidad. Así mismo, se converso y entrevistó a mujeres y hombres de la comunidad, para así entender la participación de todos los actores en la gerencia de la medicina tradicional y occidental.

Los criterios de selección de los actores en este trabajo de investigación consistió en primer lugar, que tengan conocimientos de los problemas de salud de la comunidad, que estén involucrados en el proceso salud y enfermedades (dirección, organización, agentes de salud, beneficiarios); que sean del pueblo indígena Rama y que habiten en la comunidad de Rama Cay.

Para los pueblos indígenas, la salud tiene un concepto integral, que ve al individuo en un plano individual (espiritual, físico, mental, emocional) en su medio ambiente y que a la vez cuenta con un plano comunitario (con aspectos culturales, sociales, económicos y políticos). Por tanto la participación de la comunidad indígena Rama en el campo de la salud debe percibirse en los distintos planos de su concepción del proceso salud-enfermedad. En este trabajo de investigación, se dimensionó la participación comunitaria en el plano de la salud individual y comunitaria. Para el plano de la salud individual se abordó el aspecto físico, espiritual y emocional-mental. Así mismo, en el plano de la salud comunitaria se tomo en cuenta elementos políticos, económicos, sociales y culturales.

\section{RESULTADOS Y DISCUSIÓN}

La Constitución Política de Nicaragua reconoce la multietnicidad, multiculturalidad y plurilingüismo de la nación, esta acuarela de diversidades humanas están ubicadas en su mayor parte en las Regiones Autónomas del Caribe nicaragüense, caracterizada por la 
activa lucha por la supervivencia de estos grupos humanos, unidos entre sí para tal fin por muchos siglos.

Es vertiginosa la condición de cambios en el mundo actual que afecta los cimientos de sociedades en los distintos campos del quehacer humano. Los servicios que los gobiernos procuraban a la población (salud, educación, vivienda, trabajo, etc.) han sido desplazados por empresas privadas, excluyendo a los sectores políticos, económicos, sociales y culturales más desprotegidos.

Estos mismos cambios globales también traen un lado positivo, entre otros el levantamiento de conciencias, en los procesos de apropiación de los instrumentos políticos jurídicos a nivel local, regional, nacional e internacional. En base a estas herramientas se diseñaron estrategias de intervención social, incluyendo la necesidad de una acción de base comunitaria orientada a la intervención en salud, lo que equivale a decir que la comunidad debe ser partícipe para el mejoramiento de su estado de salud.

Los objetivos que se pretenden lograr mediante la participación de la comunidad son múltiples: fortalecimiento de la estructura, movilización de recursos, la prevención más eficaz de ciertos padecimientos, adopción de hábitos y estilos de vida más saludables o la mejora de la utilización de los servicios de salud, hasta el ejercicios del derecho y el deber de participar en las decisiones que afectan la vida cotidiana, que todo ciudadano tiene. (Aguilar, s.f.)

Para alcanzar estos objetivos, en nuestro contexto encontramos los siguientes canales o mecanismos de participación: La comunidad, los grupos de auto ayuda, los Agentes Tradicionales de Salud (con el modelo de salud casero), el Ministerio de Salud, los Consejos Regionales de Salud (con participación de la Sociedad Civil), las instituciones gubernamentales y no gubernamentales.

Conocer la participación de los comunitarios de Rama Cay en salud, precisó conceptos operativos sobre la participación comunitaria en el ámbito de salud, en los planos de la salud individual y comunal.

\subsection{SAlud Individual}

En el plano de la salud individual las enfermedades de tipo biológico y físico son una prioridad. La comunidad esta conscientes de las limitaciones de medicinas en el Centro de Salud y de las alternativas que existe en el campo de la etnomedicina, así mismo, de la coexistencia de dos modelos de atención a la salud que solucionan sus distintos problemas.

Los comunitarios piensan que para mejorar la situación de salud en general de la isla, hay que poner en práctica medidas de higiene adecuadas técnicamente y la creación de leyes de salubridad de acuerdo a sus creencias e intereses como grupo. En este particular el 
Ministerio de Salud asume ser responsable, sin embargo, sus limitaciones presupuestarias hace difícil acompañar a la comunidad en este proceso.

Anteriormente, los curanderos o bush (doctor), monopolizaban el poder y fungían como intermediarios entre el mundo de los humanos y el de los espíritus, abarcando aspectos del plano de la salud comunitaria. Actualmente, la comunidad cuenta con líderes espirituales desde el ámbito religioso. Por tanto, a pesar de que el trabajo de los curanderos no tiene el poder de antes, es importante destacar que todavía se cuentan relatos sobre estos:

"Si la persona se enferma y el médico no puede hacer nada va donde el curandero. Ejemplo; una muchacha del otro lado (norte) se puso como loca, fue donde el curandero y él la curo."

Grupo focal, Rama Cay, 2002

\subsection{SAlud Individual: Emocional - Mental}

Los principales desastres que se han dado en la isla son; las inundaciones, huracanes, inviernos severos, tormentas, las sequías de los pozos y los incendios forestales. Estos desastres son de tipo natural, pero provocan conflictos emocionales (perdida de cosechas, viviendas y medios de producción). Esto aunado principalmente al sentimiento arraigado del pueblo indígena sobre la "Madre Tierra".

En la cultura Rama, las relaciones familiares son de apoyo mutuo. Esto desde luego, por la red de parentesco tan estrecho que existe en los pobladores de la isla. Por otro lado, en la comunidad las agrupaciones de mujeres con excepción de la cooperativa de mujeres, giran en torno a salud, educación y deportes, por tanto, se observa que las mujeres están participando en ocupaciones antes destinadas a los hombres, saliéndose de su rol tradicional

En la comunidad existe un proyecto productivo en el que participan mujeres con sus respectivos esposos, a pesar que no se puede denominar "exitoso" en el campo productivo (por falta de recursos e inversión), si lo es en relación a la organización comunitaria, en la que se crearon condiciones para una propuesta novedosa de trabajo compartido, equitativo y de empoderamiento a favor de las mujeres de la cooperativa.

\subsection{Plano económico de la salud comunitaria}

De acuerdo a los resultados, la situación del pueblo Rama de Rama Cay no ha sufrido grandes cambios, sus principales actividades económicas se concentran en la pesca y la agricultura de subsistencia, sin embargo, hay que destacar la escasez de estos recursos naturales, los cuales vienen a poner en peligro la sobrevivencia de los pobladores de Rama 
Cay. Los pocos ingresos obtenidos por las actividades de la pesca y la agricultura de las familias Ramas se invierten en insumos personales y domésticos.

\subsection{La Salud Comunitaria en lo político}

Los partidos políticos son los actores principales en el campo político de la comunidad. Esto desde luego, se debe al contexto nacional y regional en que vive Nicaragua y que incluye a los pueblos indígenas. Los pobladores de Rama Cay, manifiestan que la mayoría de los afiliados a estos partidos no conocen los objetivos partidarios, su pertenencia a los partidos está marcada por la simpatía más que por alcanzar aspiraciones políticas, sociales y económicas, ventaja que aprovechan los dirigentes/as de dichos partidos que no buscan reivindicaciones a favor de los indígenas.

Como consecuencia de las elecciones y de acuerdo a la Ley de Autonomía, a la comunidad Rama le corresponden dos representantes ante el Consejo Regional Autónomo como concejales. Los concejales electos son representantes de la comunidad en el ámbito regional. Existe la percepción de los habitantes, que aparte de organizar a la gente para hacer trabajos comunitarios (limpieza comunal) o cuando hay desastres, a estos le hacen falta un trabajo de mayor incidencia en la gestión de políticas publicas a favor del pueblo indígena Rama, entre estas las enfocada a servicios básicos, entre ellos la salud.

\subsection{La Salud Comunitaria en lo Social}

La Junta Directiva del Comité Comunal y su líder son la máxima instancia en la toma de decisiones en la comunidad, ya sea a nivel comunitario, familiar, personal y con los comités de la comunidad. Las familias participan en la toma de decisiones mediante su voto en las asambleas comunitarias. En la comunidad de Rama Cay, la iglesia morava juega un papel determinante en la vida social y religiosa de la zona.

Para el Ministerio de Salud y Educación representadas en la comunidad, las decisiones son tomadas por las instancias superiores regionales, por lo que la participación de la asamblea comunitaria y por ende de los líderes comunitarios no es un punto de referencia para estas instancias.

Los líderes y los comunitarios consideran que el trabajo de la junta comunal, especialmente en el sector salud es pasivo. Es decir, en relación a la salud comunitaria, la junta comunal no tienen funciones asignadas, solamente realizan actividades enfocadas hacia la limpieza comunal y jornadas de aseo. Así mismo, el apoyo del Ministerio de Salud es limitado y cerrado debido a su estructura y política de gerencia.

Los miembros de la Junta Directiva son electos por la Asamblea Comunitaria. El periodo de estos miembros es de aproximadamente dos a tres años. Los líderes comunitarios 
elegidos por medio de la asamblea comunal pueden terminar su periodo antes de tiempo por un mal funcionamiento, desinterés en la solución de los problemas e inasistencia a las reuniones o bien al tomar decisiones que atenten con la identidad y el bienestar el pueblo Rama.

Los pobladores de Rama Cay, plantean que para resolver los problemas de salud se reúnen los líderes y representantes comunitarios para analizar las diferentes situaciones y tomar decisiones. Sin embargo, curanderos y parteras, afirman sentir cierta imposición por parte del Ministerio de Salud.

\subsection{La Salud Comunitaria en lo cultural}

La comunidad de manera general asiste al centro de salud en donde los atiende personal del Ministerio de Salud. El centro de salud en Rama Cay cuenta con dos auxiliares de enfermería. Sin embargo, la comunidad también cuenta con parteras, curanderos, brigadistas, promotora, quienes coordinan con el Ministerio de Salud, y de alguna forma u otra hay una buena respuesta hacia el trabajo conjunto de atención en salud a la comunidad. En el caso particular de las mujeres embarazadas, estas prefieren a las parteras, aun cuando el Ministerio de Salud tiene programas enfocados a la atención de mujeres embazadas y los periodos de lactancia materna.

Por otro lado, es importante mencionar que las brigadas médicas del Ministerio de Salud que atienden a la población, no todos son bilingües. Esta situación de alguna forma u otra, limitan la comunicación y coordinación entre el pueblo indígena Rama y el Ministerio de Salud. Estas brigadas médicas en la mayoría de los casos el personal que lo acompaña, solamente hablan español, por lo que se necesitan traductores en la mayoría de los casos.

Concerniente a la educación, los jóvenes en la comunidad tienen pocas facilidades para estudiar. En la comunidad la educación es hasta secundaria. Así mismo, es importante mencionar que como parte del proceso de revitalización de la lengua Rama, se imparten clases en la lengua Rama en el centro de estudio de la comunidad.

En el caso de las mujeres de la Asociación de Mujeres Indígenas Ramas, la asociación representó un grupo organizado para crear espacios de participación propia de las mujeres.

\section{CONCLUSIONES}

- La participación de la comunidad en el desarrollo sanitario está enmarcada en la atención primaria de salud, en este contexto interactúan: la comunidad y sus agentes de salud, los ONG's, los profesionales y el Ministerio de Salud. El 
papel de este último es disponer de mecanismos que permitan la participación institucionalizada, real y efectiva de la comunidad, de sus agentes tradicionales de salud y los profesionales de la salud como los protagonistas que interactúan en la búsqueda de soluciones y alternativas para mejorar el estado de salud de la mayoría de las personas de manera armoniosa.

- El liderazgo comunitario es un factor potenciador de la participación comunitaria, que impulsa el alcance de los objetivos, moviliza a los beneficiarios, establecen formas de participación tradicional, etc. Los sistemas de apoyo mutuo en el ámbito de la salud no es nuevo en la comunidad, también se aplican en los distintos planos de la salud comunitaria. Otros factores que potencian la participación están asociados al concepto de comunidad como la identidad del pueblo indígena Rama, para los comunitarios la participación por el grupo y para la comunidad es un estímulo.

- Un beneficio de la participación social en las Regiones Autónomas es promovido en las comisiones municipales de salud (Instancias municipales de consulta y asesoría en salud) y los Consejos Regionales de Salud (instancia regional de asesoría en salud al Consejo Regional Autónomo) a nivel regional. Sin embargo a pesar de que el pueblo Rama no tiene presencia en estas instancias, estas representan un potencial espacio para el desarrollo de un modelo de salud para este pueblo indígena.

- Para el Ministerio de Salud, la participación de la comunidad debe presentarse desde los canales establecidos como: los programas de apoyo mutuo, los brigadistas y promotores de salud.

- La universidad URACCAN tiene en su propuesta de participación comunitaria una relación más directa, es una universidad comunitaria que abre las puertas a estudiantes de la comunidad con becas estudiantiles, Interactúan mediante sus docentes y estudiantes en investigaciones en y con la comunidad, tiene programas concretos para las comunidades y pueblos indígenas (rescate cultural, educación intercultural bilingüe, diplomados de desarrollo comunitario, etc) y formación de liderazgo.

- Acción Médica Cristiana (ONG), enfoca su estrategia de intervención en la participación de los comunitarios Ramas como beneficiarios de talleres, en el intercambio de conocimientos con los Agentes Tradicionales de Salud y con una promotora de la comunidad que coordina trabajos con los auxiliares de enfermería. 
- Los pueblos indígenas conciben la participación por medio de espacios institucionalizados y tradicionales donde intervienen la asamblea comunal $^{19}$ y las autoridades comunales tradicionales ${ }^{20}$. En la Ley 445, art. 3 la institución correspondiente concibe la participación mediante la consulta definida como "la expresión y entrega de información técnica de la operación o el proyecto, seguido de un proceso de discusión y decisión sobre los mismos; durante los cuales las comunidades deberán contar con traductores, los que traducirán en sus lenguas todo lo dicho durante este proceso y estar asistidas por técnicos en la materia. Tanto el traductor como los técnicos deberán ser escogidos y nombrados por las comunidades ${ }^{21}$ ".

\section{RECOMENDACIONES}

- Los beneficios que se obtienen de la participación comunitaria deben caracterizar al sistema de salud, se debe ampliar los canales de participación de las instituciones (gubernamentales o no), debe reflejarse también el involucramiento de actores comunitarios que desarrollen conciencia en su derecho y el deber que tienen de participar, tener presencia de comunitarios Ramas en los Consejos Regionales de Salud.

- Se debe diseñar un Modelo de salud tomando en cuenta elementos como el uso de medicina tradicional, cosmovisión, particularidades organizativas y de toma de decisión, formas de comunicación, participación comunitaria en salud, prácticas médicas, accesibilidad, relaciones entre agentes de salud y preferencias de servicios médicos. Así mismo, este modelo debe garantizar el seguimiento por medio de un plan de monitoreo y evaluación que intege a los comunitarios.

\section{VII.BIBLIOGRAFÍA}

Aguilar Idánez, Maria José. s.f. Participación Ciudadana en Salud: Evaluación de Experiencias. p.irr. Managua, Nicaragua.

Asamblea Nacional de Nicaragua. 1995. Estatuto de Autonomía para las regiones de la costa Atlántica. Managua, Nicaragua.

19 Es la reunión de los miembros de la comunidad, congregados para tomar decisiones sobre asuntos de interés comunitario, de conformidad con sus costumbres y tradiciones.

20 Es la autoridad de la comunidad indígena y étnica, elegida en Asamblea Comunal según sus costumbres y tradiciones para que lo represente y los gobierne; tales como el Síndico, Whita, Coordinador u otros.

21 Asamblea Nacional de la República de Nicaragua. 2002 en; Ley del Régimen de Propiedad Comunal de los Pueblos Indígenas y Comunidades Étnicas de las Regiones Autónomas de la Costa Atlántica de Nicaragua y de los Ríos Bocay, Coco, Indio Y Maíz. 
Asamblea Nacional de Nicaragua. 2002. Constitución Politica. Edit. Parlamento, Managua, Nicaragua.

Asamblea Nacional de Nicaragua. 2002. Ley de Régimen de la Propiedad Comunal de los Pueblos Indígenas y Comunidades Étnicas de las Regiones Autónomas de la Costa Atlántica de Nicaragua y de los Ríos Bocay, Coco, Indio y Maiz. P. Irr.

Conferencia Internacional del grupo consultivo sobre la reconstrucción y Transformación de América Central. 1999. Problemática y Desafios de la Sociedad de la Costa Caribe de Nicaragua y la propuesta de las estrategias regionales de desarrollo sostenible. Estocolmo Suecia. S n t .NI 24 P

González Miguel, 2001. Desarrollo humano en la Costa Caribe de Nicaragua.

CONPES - PNUD- PRANAF. Managua, Nicaragua.--/ 63 PG.

McGee Rosemery, 2002. Assesing Participation in Poverty Strategy papers: a desk-based synthesis of experience in Sub-Sahara Africa.Institute of Development Studies. Brighton, Sussex BNI 9RE. England.

Mejía Mario, Cruz Rosa, 2002. Elementos claves para un modelo de atención en salud para la comunidad indígena de Rama Cay, Bluefields. Febrero-Julio del 2002. Universidad de las Regiones Autónomas de la Costa Caribe de Nicaragua. Bluefields, RAAS.86 p.

Proyecto de Ley General de Salud de la República de Nicaragua. 2002

Managua, NI. 11 p.

UNOPS-UNDP, 1999._Autonomous Regions of the Atlantic Coast of Nicaragua. P. irr. 